\title{
Effect of Ambient Wind Velocity on Planetary Nebula Morphology
}

\author{
Vikram V. Dwarkadas $^{1,2}$, Roger A. Chevalier ${ }^{1}$ and John M. Blondin ${ }^{3}$ \\ ${ }^{1}$ University of Virginia, Charlottesville; ${ }^{2}$ University of Washington, Seattle; \\ ${ }^{3}$ North Carolina State University, Raleigh
}

Planetary Nebulae (PNe) are formed by the interaction of the fast wind from a post-Asymptotic Giant Branch Star with the slow ambient wind from a previous epoch. If the two interacting winds have constant properties, the velocity of the PN shell tends towards a constant with time and the shape becomes self-similar. Additionally, if the velocity of the fast wind is much higher than the expansion velocity of the shell, the interior of the hot shocked bubble becomes isobaric. Using semi-analytical methods, complemented by hydrodynamic simulations, we have calculated the shapes of PNe in the self-similar stage (Dwarkadas et al. 1996). We have investigated the contribution of the ambient wind velocity to PN morphology, which has hitherto not received much attention since the work of Kahn \& West (1985). We find that the nebular morphology is a consequence of the density contrast between pole and equator in the ambient medium, the steepness of the density profile and the velocity of the ambient wind; classification of PNe purely on the basis of the first two factors may be misleading. In particular, the ratio of ambient wind velocity to PN velocity is important in determining whether the nebula shows a bulge or a cusp at the equator. A high density contrast coupled with a low velocity for the external medium gives rise to extremely bipolar nebulae. For large density contrasts and a significant value of the slow wind velocity, the surface density maximum of the shell shifts away from the equator, giving rise to peanut-shaped structures with pronounced equatorial bulges. As long as the external wind velocity is small compared to the expansion velocity of the nebula, the PNe tend to be more bipolar, even with a moderate density contrast. If the PN velocity is close to that of the external wind, the shape is relatively spherical. However, inclusion of an asymmetric velocity profile in the slow wind, with the velocity increasing towards the pole, can lead to a bipolar nebula if the equatorial velocity is sufficiently low. Preliminary results with a slow wind velocity increasing towards the equator (as is found in calculations of common envelope evolution) show that the nebulae tend to be more oblate, which is not often observed in nature. Representative results for shapes of PNe using various values of the relevant parameters are presented.

I gratefully acknowledge award of an International Travel Grant from the American Astronomical Society, as well as a travel grant from the International Astronomical Union, which enabled me to attend the meeting.

\section{REFERENCES}

Dwarkadas, V. V, Chevalier, R. A., \& Blondin, J. M., 1996, Astrophy. J., 457, 773

Kahn, F. D., \& West, K. A., 1985, Mon. Not. Royal Ast. Soc., 212, 837 